02,09

\title{
Параметрическое усиление высокочастотного излучения точечными контактами Джозефсона вблизи субгармонических ступенек Шапиро
}

\author{
(C) О.Д. Позднякова, А.В. Шорохов \\ Мордовский государственный университет им. Н.П. Огарева, \\ Саранск, Россия \\ E-mail: alex.shorokhov@mail.ru
}

Поступила в Редакцию 16 сентября 2018 г.

В окончательной редакции 16 сентября 2018 г.

Принята к публикации 31 января 2019 г.

В рамках резистивной модели изучено параметрическое усиление высокочастотного излучения точечными контактами Джозефсона в резонаторе. Численно и аналитически показано, что вблизи субгармонических ступенек вольт-амперной характеристики имеются области усиления электромагнитного излучения. Рассмотрен одномерный массив последовательно соединенных точечных контактов Джозефсона. Показано, что в массиве контактов вблизи субгармонических ступенек вольт-амперной характеристики контакты не синхронизируются и, соответственно, области усиления вблизи субгармонических ступенек для массива отсутствуют.

DOI: $10.21883 /$ FTT.2019.06.47678.290

\section{1. Введение}

Известно, что если ток через джозефсоновский контакт превышает некоторое критическое значение, обусловленное свойствами самого контакта, на контакте возникает разность потенциалов, что приводит к нестационарному эффекту Джозефсона [1]. Частота джозефсоновского излучения может достигать нескольких сотен $\mathrm{GHz}$. Вблизи критической температуры для анализа высокочастотных (ВЧ) явлений удобно использовать резистивную модель [2], согласно которой джозефсоновский контакт может быть представлен в виде параллельного соединения идеального джозефсоновского контакта, через который течет только бездиссипативный ток куперовских пар, и резистивного элемента с активным сопротивлением, через который течет диссипативный ток одиночных электронов проводимости (нормальный ток). Для учета емкости контакта в резистивной модели в схему добавляют также конденсатор. Для некоторых типов джозевсоновских контактов (например, для точечных контактов) емкость можно не учитывать, так как она очень мала. Контакты с малой емкостью легче согласовывать с внешними электродинамическими системами СВЧ [3].

В рамках резистивной модели и с помощью теории возмущений в [4-7], а также с использованием численных методов [8] было показано, что во внешнем переменном поле действительная часть импеданса контакта может принимать отрицательные значения при частотах внешнего излучения, близких к частоте джозефсоновской генерации, причем частота внешнего сигнала должна быть больше частоты джозефсоновской генерации. В $[4,5]$ в слабосигнальном приближении было показано, что аномальное поведение импеданса контакта Джозефсона обусловлено поведением индуктивности контакта. Таким образом, теоретически предсказывалась возможность параметрического усиления внешнего высокочастотного излучения, при этом предполагалось использовать в качестве накачки джозефсоновское излучение. Аномальное поведение импеданса и связанное с ним параметрическое усиление экспериментально наблюдалось в [9] и [10]. Благодаря параметрическому взаимодействию джозефсоновского и внешнего ВЧ излучения на контакте, частоту усиливаемого сигнала можно сдвинуть, в том числе, и в $\mathrm{THz}$ диапазон.

Мощность параметрического (неджозефсоновского) излучения одиночным джозефсоновским контактом очень мала (порядка нескольких $\mathrm{nW}$ ). В связи с этим большой интерес представляют массивы синхронизированных контактов Джозефсона, способных излучать когерентно. Мощность параметрического излучения, полученная с помощью больших массивов джозефсоновских контактов, может достигать нескольких $\mu \mathrm{W}[11,12]$.

\section{2. Одиночный контакт Джозефсона}

Параметрическое усиление внешнего ВЧ излучения одиночным контактом Джозефсона было хорошо изучено теоретически и экспериментально, как было отмечено ранее. Для лучшего понимания полученных ниже результатов коротко рассмотрим одиночный контакт Джозефсона без резонатора.

При $T \approx T_{c}$ полный ток через джозефсоновский контакт можно представить в виде $I(t)=I_{J}+I_{N}$ $=I_{c} \sin (\varphi)+V / R_{J}$, где $I_{J}=I_{c} \sin (\varphi)$ - джозефсоновский ток, $I_{c}-$ критический ток, $\varphi-$ разность фаз 
контактирующих сверхпроводников, $I_{N}-$ нормальный ток, $V=I_{N} R_{J}$ - напряжение на контакте, $R_{J}-$ сопротивление контакта в нормальном состоянии [2]. Эквивалентная схема контакта представлена на рис. 1 .

Действие внешнего электромагнитного излучения частоты $\omega_{1}$ приведет к появлению дополнительного переменного тока через контакт. Тогда полный ток $I(t)=I_{d c}+I_{1} \cos \left(\omega_{1} t\right)$, где $I_{d c}-$ постоянный ток. Учитывая связь между напряжением на контакте и разностью фаз $\frac{d \varphi}{d t}=\frac{2 e}{\hbar} V(t)$, из резистивной модели получим уравнение для разности фаз:

$$
\frac{d \varphi}{d \tau}+\sin (\varphi)=i_{d c}+i_{1} \cos \left(\Omega_{1} \tau\right)
$$

где $\tau=\omega_{c} t, \omega_{c}=\frac{2 e}{\hbar} V_{c}-$ характерная частота контакта, $V_{c}=I_{c} R_{J}$ - характерное напряжение, $i_{d c}=\frac{I_{d c}}{I_{c}}, i_{1}=\frac{I_{1}}{I_{c}}$, $\Omega_{1}=\frac{\omega_{1}}{\omega_{c}}$. Уравнение (1) представляет собой уравнение

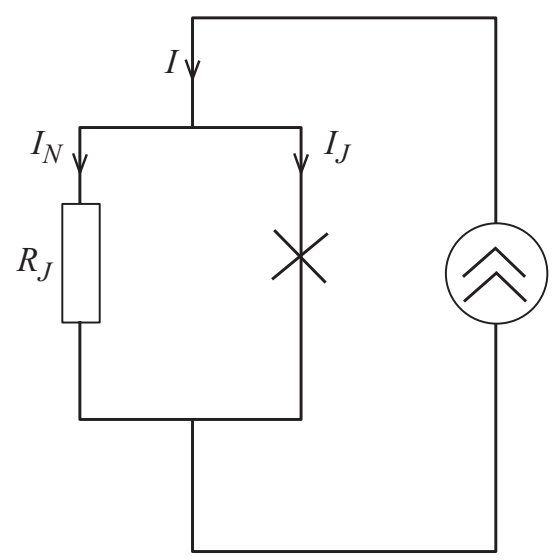

Рис. 1. Эквивалентная схема контакта Джозефсона без учета внутренней емкости.
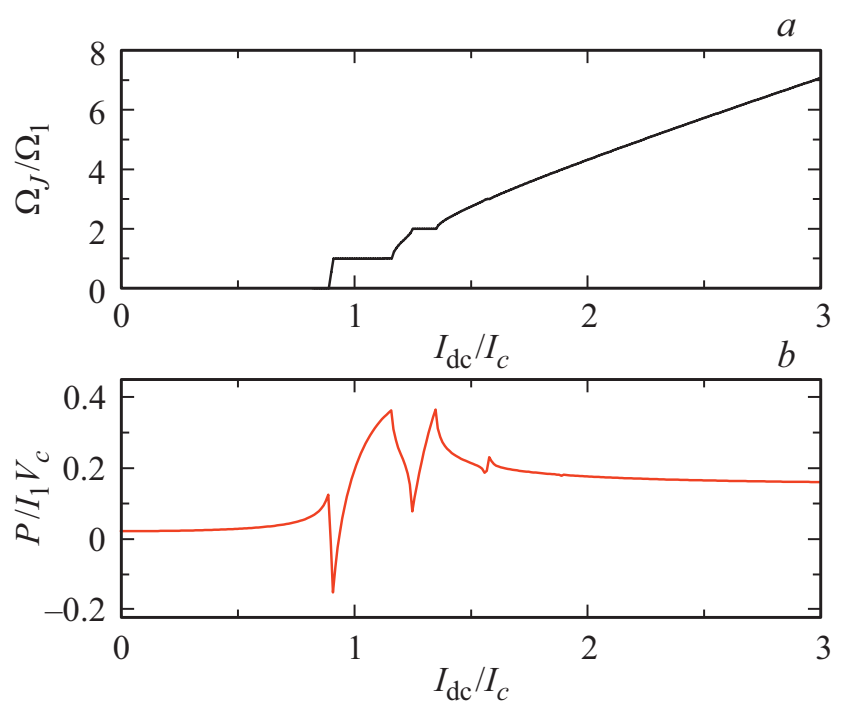

Pис. 2. a) ВАХ джозефсоновского контакта и $b$ ) график зависимости поглощенной мощности от постоянного тока для частоты $\omega_{1}=0.4 \omega_{c}$ и амплитуды переменного тока $I_{1}=0.3 I_{c}[8]$.

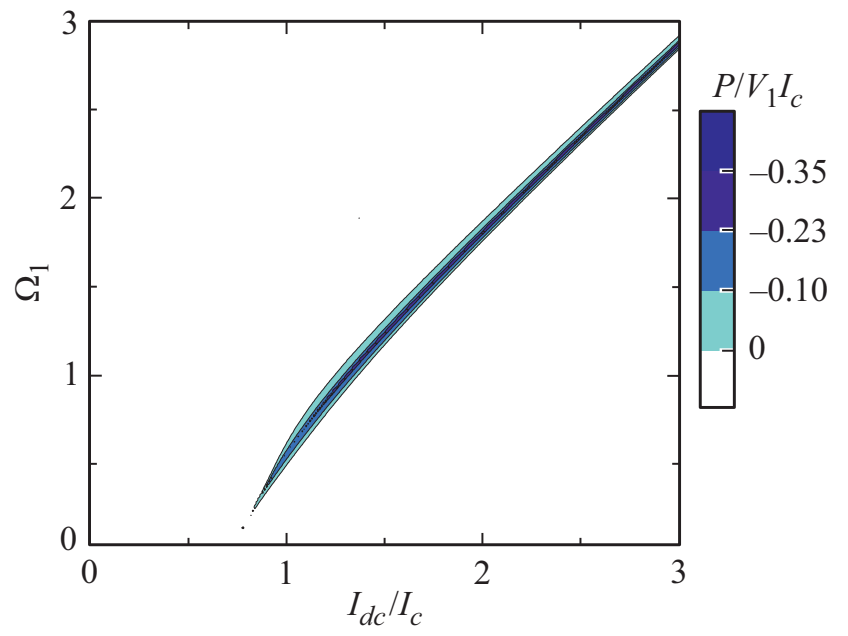

Рис. 3. Средняя поглощенная мощность одиночного точечного контакта Джозефсона. $I_{1}=0.3 I_{c}$.

нелинейного передемпфированного маятника. Известно, что действие внешнего высокочастотного излучения на контакт Джозефсона приводит к появлению ступеней тока на BAX контакта (ступеньки Шапиро [13]) (см. рис. 2).

Среднюю поглощенную мощность внешнего высокочастотного излучения на его частоте можно найти по формуле

$$
\frac{P}{I_{1} V_{c}}=\left\langle\dot{\varphi} \cos \left(\Omega_{1} \tau\right)\right\rangle_{\tau}
$$

BAX и график зависимости поглощенной мощности от постоянного тока представлены на рис. 2.

Средняя поглощенная мощность, расчитанная по формуле (2), с точностью до постоянного множителя пропорциональна действительной части импеданса, численно найденного в работе [8]. Области усиления для различных значений постоянного тока $I_{d c}$ и частоты пробного тока $\Omega_{1}$ показаны на рис. 3 .

На рис. 3 видим лишь одну полосу усиления, которая для всех значений частот $\Omega_{1}$ и токов $I_{d c}$ соответствует первой ступеньке Шапиро.

\section{3. Одиночный контакт Джозефсона в резонаторе}

Рассмотрим контакт Джозефсона в резонаторе. Резонатор представим как $L C$-контур (рис. 4).

Для данной системы можно записать следующие уравнения

$$
\left\{\begin{array}{l}
\frac{\hbar}{2 e R_{J}} \frac{d \varphi}{d t}+I_{c} \sin (\varphi)+\frac{d Q}{d t}=I_{d c}+I_{1} \cos \left(\omega_{1} t\right), \\
L \frac{d^{2} Q}{d t^{2}}+\frac{Q}{C}=\frac{\hbar}{2 e} \frac{d \varphi}{d t},
\end{array}\right.
$$

где $Q-$ заряд на обкладках конденсатора; $L-$ индуктивность резонатора; $C$ - емкость резонатора. 


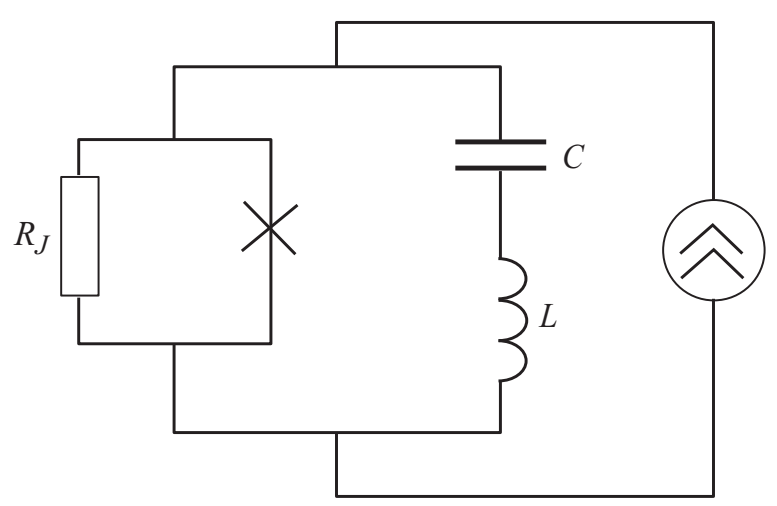

Рис. 4. Контакт Джозефсона в резонаторе.

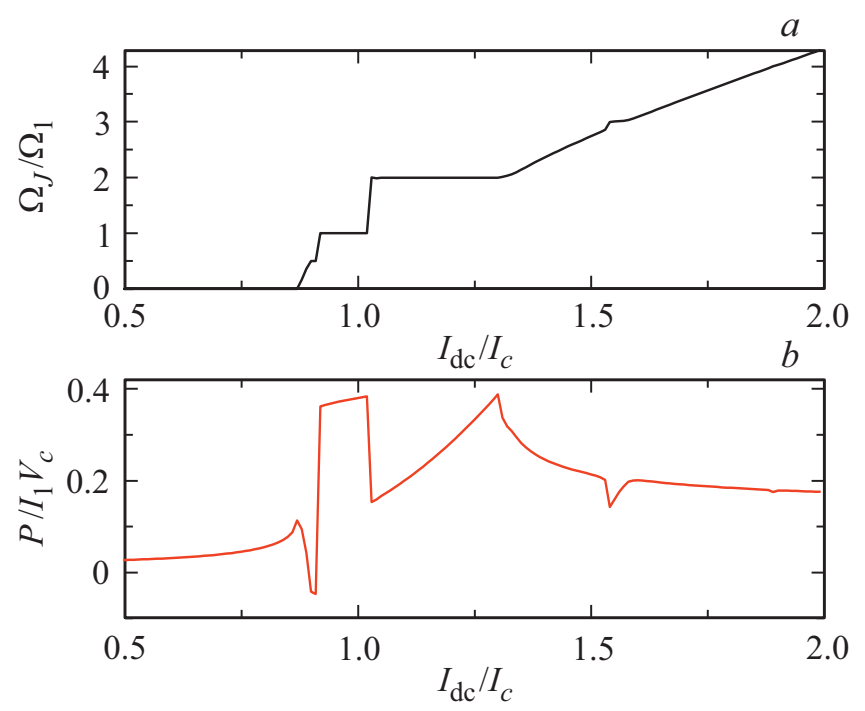

Рис. 5. $a)$ ВАХ и $b$ ) поглощенная мощность контакта в резонаторе при $\Omega_{1}=0.4 ; \Omega_{0}=0.8 ; I_{1}=0.3 I_{c} ; \beta=0.01$.
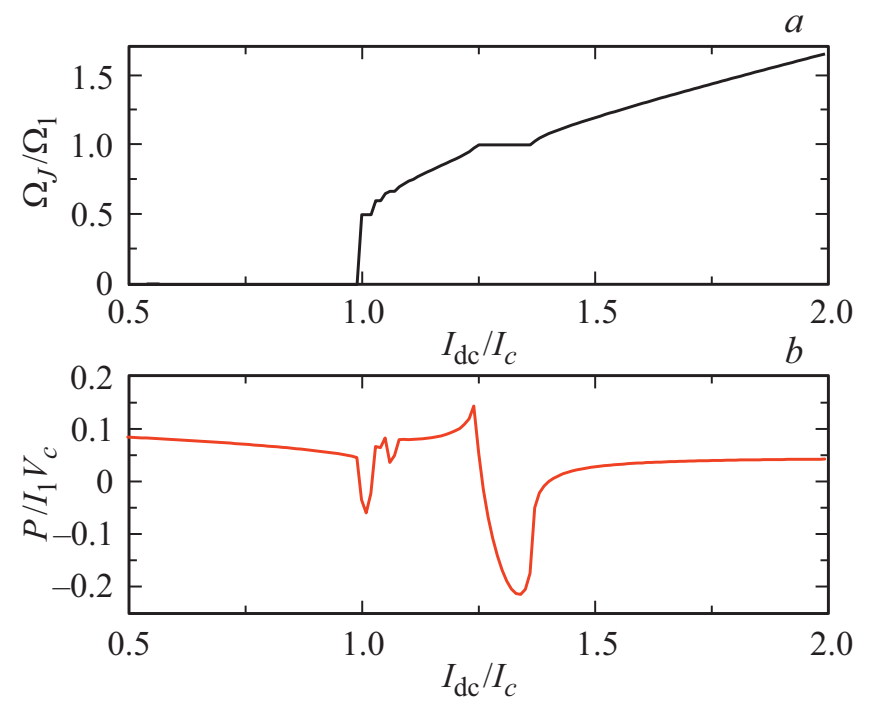

Рис. 6. $a)$ ВAX и $b$ ) поглощенная мощность контакта в резонаторе при $\Omega_{1}=1.2 ; \Omega_{0}=2.4 ; I_{1}=0.3 I_{c} ; \beta=1$.
Перейдя в системе уравнений (3) к безразмерным величинам, получим

$$
\left\{\begin{array}{l}
\dot{\varphi}+\sin (\varphi)+\beta \dot{q}=i_{d c}+i_{1} \cos \left(\Omega_{1} \tau\right), \\
\ddot{q}+\Omega_{0}^{2} q=\Omega_{0}^{2} \dot{\varphi}
\end{array}\right.
$$

где $q=\frac{Q}{R_{J} I_{c} C} ; \Omega_{0}=\frac{1}{\sqrt{l \beta}} ; l=\frac{L}{L_{c}} ; L_{c}=\frac{\hbar}{2 e I_{c}} ; \beta=\frac{2 e}{\hbar} R_{J}^{2} I_{c} C$; $\dot{\varphi}$ и $\ddot{q}-$ первая и вторая производные по $\tau=\omega_{c} t$ соответственно.

Решив численно систему уравнений (4), можно найти среднюю поглощенную мощность по формуле (2). Численный анализ показал, что при $\Omega_{0}=n \Omega_{1}$, где $n=1,2,3 \ldots$, амплитуда тока в резонаторе возрастает [14]. Однако, если $\Omega_{0}=\Omega_{1}$, то колебания тока в резонаторе и колебания $\dot{\varphi}$ происходят с джозефсоновской частотой, а не с частотой внешнего сигнала $\Omega_{1}$, и среднее значение поглощенной мощности стремится к нулю.

Более интересен случай, когда $\Omega_{0}=n \Omega_{1}$, где $n>1$. В этом случае на ВАX контактах могут возникать субгармонические ступеньки Шапиро и отрицательная поглощенная мощность (т.е. усиление внешнего сигнала) может возникать не только, когда $\Omega_{J}=\Omega_{1}$ (первая ступенька Шапиро), но и при $\Omega_{J}=\frac{\Omega_{1}}{2}$ (субгармонические ступеньки), как видно из рис. 5 и 6 . Если из схемы на рис. 4 исключить катушку индуктивности и устремить к нулю индуктивность в уравнениях (3), то, очевидно, мы получим задачу для одиночного джозефсоновского перехода с конечной внутренней емкостью во внешнем ВЧ поле. Для переходов с внутренней емкостью могут наблюдаться субгармонические ступеньки на BAX [15-20].

На рис. 7 и 8 представлены области усиления для одиночного контакта в резонаторе для $\beta=0.01$ и $\beta=1$ соответственно.

Из сравнения рис. 3 и 7 видно, что для малых емкостей резонатора $(\beta=0.01)$ области усиления для контакта без резонатора и в резонаторе имеют небольшие различия лишь в области небольших частот $\left(\Omega_{1}<1\right)$, где первая ступенька Шапиро может быть сдвинута относительно $I_{d c}=I_{c}$ в сторону меньшего значения тока. Из сравнения рис. 3 и 8 видно, что при достаточно больших емкостях резонатора $(\beta=1)$ области усиления могут появиться вблизи субгармонических ступенек (маленькие голубые области на рис. 9, находящиеся отдельно от основной области усиления).

Рассмотрим подробнее случай малой емкости резонатора $(\beta \ll 1)$. Численный анализ показал, что при $\beta \ll 1$ в системе уравнений (4) ток через резонатор $\tilde{i}_{r}=\beta \dot{q}$ является величиной того же порядка, что и внешний слабый ток $\tilde{i}_{1}=i_{1} \cos \Omega_{1} \tau$. Таким образом, ток $\tilde{i}=\tilde{i}_{1}-\tilde{i}_{r}$ в первом уравнении системы (4) можно считать слабым возмущением и линеаризовать систему уравнений (4) так же, как сделано авторами работы [4] для контакта Джозефсона без резонатора. 
Пусть наличие слабого возмущения тока $\tilde{i}$ приводит к слабому возмущению разности фаз на контакте $\tilde{\varphi}$. Тогда разность фаз контактирующих сверхпроводников можно записать в виде $\varphi=\varphi^{(0)}+\tilde{\varphi}$, где $\varphi^{(0)}-$ решение невозмущенного уравнения

$$
\dot{\varphi}^{(0)}+\sin \varphi^{(0)}=i_{d c} .
$$

Решение уравнения (5) было получено в работе [4].

В результате линеаризации (4) получим следующую систему линейных дифференциальных уравнений

$$
\left\{\begin{array}{l}
\dot{\tilde{\varphi}}+\tilde{\varphi} \cos \left(\varphi^{(0)}\right)=\tilde{i}_{1}-\beta \dot{q}, \\
\ddot{q}+\Omega_{0}^{2} q=\Omega_{0}^{2} \dot{\varphi}^{(0)} .
\end{array}\right.
$$

Второе уравнение системы (6) перепишем с учетом полученного в [4] выражения для $\varphi^{(0)}$ в виде

$$
\ddot{q}+\Omega_{0}^{2} q=\frac{\Omega_{0}^{2} \Omega_{J}^{2}}{\sqrt{1+\Omega_{J}^{2}}+\cos \Omega_{J} \tau} .
$$

Решение системы (6) приведено в приложении А.

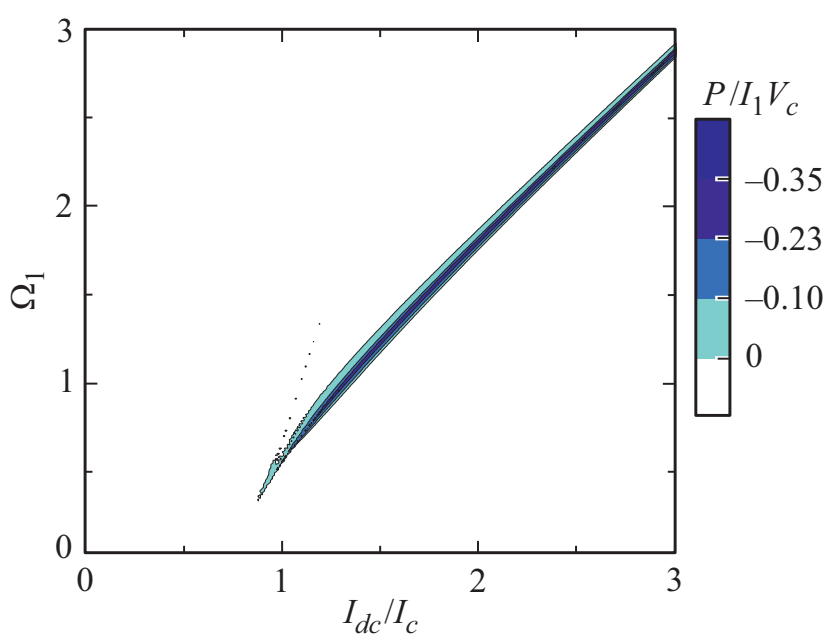

Рис. 7. Средняя поглощенная мощность одиночного точечного контакта Джозефсона в резонаторе при $\beta=0.01 . I_{1}=0.3 I_{c}$.

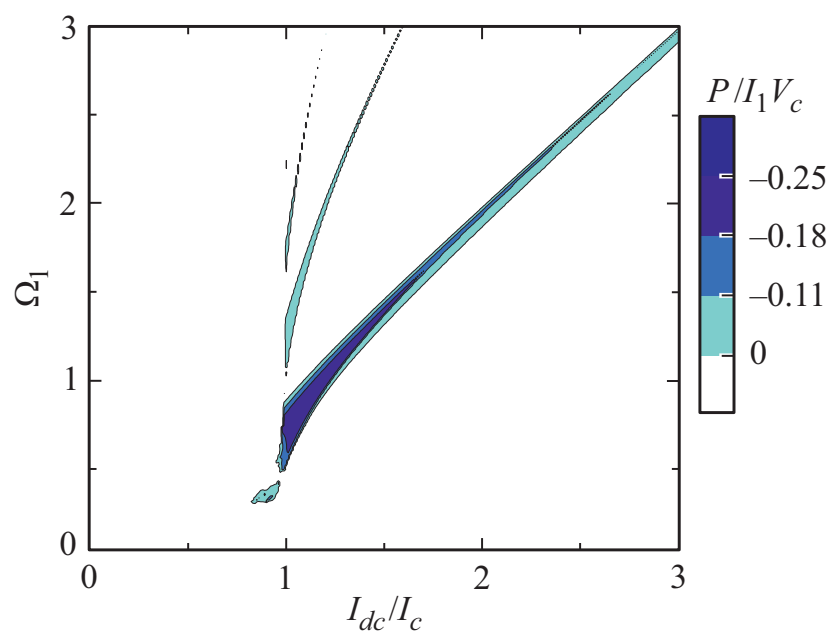

Pис. 8. Средняя поглощенная мощность одиночного точечного контакта Джозефсона в резонаторе при $\beta=1 . I_{1}=0.3 I_{c}$.
Из системы (6) можно записать выражение для отклика напряжения на контакте

$$
\dot{\tilde{\varphi}}=\tilde{i}_{1}+\ddot{\varphi}^{(0)} \int \frac{\tilde{i}_{1}}{\dot{\varphi}^{(0)}} d \tau-\left(\beta \dot{q}+\ddot{\varphi}^{(0)} \int \frac{\beta \dot{q}}{\dot{\varphi}^{(0)}} d \tau\right)
$$

Введем обозначения:

$$
\begin{gathered}
\dot{\tilde{\varphi}}_{1}=\tilde{i}_{1}+\ddot{\varphi}^{(0)} \int \frac{\tilde{i}_{1}}{\dot{\varphi}^{(0)}} d \tau ; \\
\dot{\tilde{\varphi}}_{r e s}=-\left(\beta \dot{q}+\ddot{\varphi}^{(0)} \int \frac{\beta \dot{q}}{\dot{\varphi}^{(0)}} d \tau\right) .
\end{gathered}
$$

Выражение для $\dot{\tilde{\varphi}}_{1}$ ничто иное, как уравнение для отклика напряжения на контакте, полученное в работе [4], а выражение для $\dot{\tilde{\varphi}}_{\text {res }}$ аналогично ему, но включает вместо внешнего слабого сигнала произведение тока в резонансном контуре на малый параметр емкости резонатора $\beta \dot{q}$.

Возьмем слабый внешний сигнал в виде $\tilde{i}_{1}=$ $=\exp \left(i \Omega_{1} \tau\right)$. Найдем действительную часть импеданса $\operatorname{ReZ}$ для точечного контакта в резонаторе с малой емкостью как

$$
\operatorname{Re} Z=\left\langle\frac{\dot{\tilde{\varphi}}}{\tilde{i}_{1}}\right\rangle=\left\langle\operatorname{Re} \frac{\dot{\tilde{\varphi}}_{1}}{\tilde{i}_{1}}\right\rangle+\left\langle\operatorname{Re} \frac{\dot{\tilde{\varphi}}_{\text {res }}}{\tilde{i}_{1}}\right\rangle=\operatorname{Re} Z_{1}+\operatorname{Re} Z_{r e s},
$$

где введены обозначения:

$$
\begin{gathered}
\operatorname{Re} Z_{1}=\left\langle\operatorname{Re} \frac{\dot{\tilde{\varphi}}_{1}}{\tilde{i}_{1}}\right\rangle \\
\operatorname{Re} Z_{r e s}=\left\langle\operatorname{Re} \frac{\dot{\tilde{\varphi}}_{r e s}}{\tilde{i}_{1}}\right\rangle .
\end{gathered}
$$

и

Выражение для $\operatorname{Re} Z_{1}$ получено в [4]. Выражение для $\operatorname{Re} Z_{r e s}$ получено в приложении А. В приложении А показано, что среднее значение $\left\langle\operatorname{Re} \frac{\dot{\tilde{\varphi}}_{1}}{\dot{i}_{1}}\right\rangle$ отлично от нуля лишь в случае кратных частот $\left(\Omega_{0}, \Omega_{J}\right.$ и $\left.\Omega_{1}\right)$. Рассмотрим $\Omega_{0}=\Omega_{1}=k \Omega_{J}$ при $k=2,3, \ldots$ (не рассматриваем $k=1$, т. к. при $k=1$ основной вклад дает $\left.\operatorname{Re} Z_{1}\right)$. В этом случае для $\operatorname{Re} Z_{\text {res }}$ получено:

$$
\begin{aligned}
\operatorname{Re} Z_{r e s}= & -\beta k^{2} \Omega_{J}^{2}\left(\Omega_{J}-\sqrt{1+\Omega_{J}^{2}}\right)^{k} \\
& \times\left[\Omega_{J}+\frac{\Omega_{J}-\sqrt{1+\Omega_{J}^{2}}}{\left(k^{2}-1\right) \Omega_{J}}\right] .
\end{aligned}
$$

Из (14) видно, что $\operatorname{Re} Z_{r e s}<0$ либо при нечетных $k$ и $\Omega_{J}<1$ (в этом случае выражение в квадратных скобках отрицательное), либо при четных $k$ и $\Omega_{J} \geq 1$ (выражение в квадратных скобках положительное).

Теперь рассмотрим соотношение частот $\Omega_{0}=n \Omega_{1}$ $=k \Omega_{J}$, где $n=2,3 \ldots$ и $k>1$. В этом случае получим 
следующее выражение для $\operatorname{Re} Z_{\text {res }}$ :

$$
\begin{aligned}
& \operatorname{Re}_{r e s}=\frac{1}{2} \beta \Omega_{0}^{2} \frac{\left(\Omega_{J}-\sqrt{1+\Omega_{J}^{2}}\right)^{2 k-p-1}}{\Omega_{0}+k \Omega_{J}} \\
& \quad \times\left[4(k-p) \sqrt{1+\Omega_{J}^{2}}\left(\Omega_{J}-\sqrt{1+\Omega_{J}^{2}}\right)\right. \\
& +\frac{(k-p+1) \Omega_{0}}{\Omega_{J}+\Omega_{0}}\left(\Omega_{J}-\sqrt{1+\Omega_{J}^{2}}\right)^{2}+\frac{(k-p-1) \Omega_{0}}{\Omega_{0}-\Omega_{J}} \\
& +\frac{(k+p+1) \Omega_{0}}{\Omega_{J}+\Omega_{0}}\left(\Omega_{J}-\sqrt{1+\Omega_{J}^{2}}\right)^{2(p+1)} \\
& \left.+\frac{(k+p-1) \Omega_{0}}{\Omega_{0}-\Omega_{J}}\left(\Omega_{J}-\sqrt{1+\Omega_{J}^{2}}\right)^{2 p}\right]
\end{aligned}
$$

где $p=\frac{k}{n}$ - целое число, такое, что $p>1$ и $\Omega_{1}=p \Omega_{J}$.

$\mathrm{B}(15)$ можно подобрать такие $k, n$ и $\Omega_{J}$, что выражение в скобках будет положительным. Тогда $\operatorname{Re} Z_{r e s}<0$, если $(2 k-p-1)-$ нечетное. Это соответствует соотношению частот $\Omega_{J}=\frac{\Omega_{1}}{p}$, где $p-$ четное. Также, если подобрать такие $k, n$ и $\Omega_{J}$, что выражение в скобках будет отрицательным, то $\operatorname{Re} Z_{\text {res }}<0$ при условии, что $(2 k-p-1)$ - четное; в этом случае $p-$ нечетное. Области усиления вблизи субгармонических ступенек BAX были найдены и при численном анализе системы уравнений (4), что было отмечено выше.

\section{4. Массив джозефсоновских контактов}

Существуют различные способы соединения джозефсоновских контактов в массив и различные механизмы и модели их синхронизации [21-24]. Мы изучали одномерный массив последовательно соединенных точечных контактов Джозефсона в резонаторе.

Массив джозефсоновских контактов можно представить как систему связанных нелинейных осцилляторов. Такая система может находиться в следующих состояниях: 1) колебания всех осцилляторов согласованы по фазе (coherent state); 2) согласование отсутствует (incoherent state). В свою очередь возможны два вида coherent state: 1) колебания всех осцилляторов происходят в

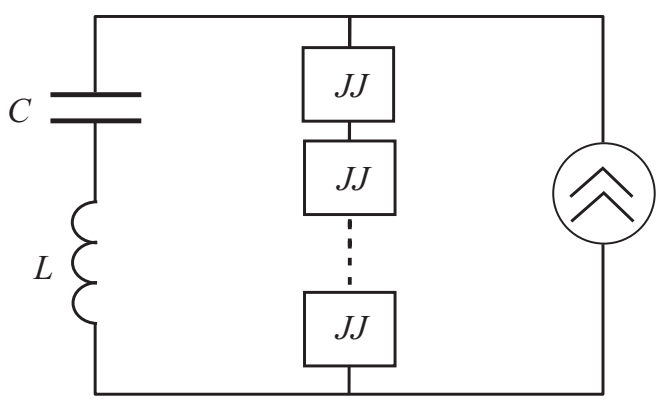

Рис. 9. Массив $N$ одинаковых контактов в резонаторе. одинаковой фазе (in-phase state), т. е. $\varphi_{1}=\varphi_{2}=\ldots=\varphi_{N}$ для любого момента времени; 2) колебания всех осцилляторов происходят с одинаковой разностью фаз (splay state), т. е. $\varphi_{1}(t)=\varphi_{2}\left(t+\frac{T}{N}\right)=\ldots=\varphi_{N}\left(t+\frac{N-1}{N} T\right)$ для любого момента времени [25]. Для того, чтобы было усиление внешнего излучения массивом контактов, контакты в массиве должны быть синхронизированы. Наиболее оптимальным для усиления является режим in-phase. Стабильность синхронизированного состояния как для одномерного, так и для двумерного массива была проанализирована с помощью теории Флоке в многочисленных работах [26-30]. Для одномерного массива последовательно соединенных контактов с пренебрежимо малой внутренней емкостью синхронизированное состояние (in-phase state) стабильно при индуктивной внешней нагрузке, а при емкостной -- нестабильно [27].

Рассмотрим массив $N$ одинаковых контактов в резонаторе, как показано на рис. 9.

Эквивалентная схема каждого джозефсоновского контакта $(J J)$ представлена на рис. 1.

Поместим массив контактов во внешнее высокочастотное излучение. Зафиксируем полный ток в виде $I(t)=I_{d c}+I_{1} \cos \left(\omega_{1} t\right)$. В безразмерных единицах получим следующую систему $N+1$ уравнений:

$$
\left\{\begin{array}{l}
\dot{\varphi_{k}}+\sin \left(\varphi_{k}\right)+\beta \dot{q}=i_{d c}+i_{1} \cos \left(\Omega_{1} \tau\right), \quad k=1,2, \ldots, N ; \\
\ddot{q}+\Omega_{0}^{2} q=\Omega_{0}^{2} \sum_{k=1}^{N} \dot{\varphi}_{k} .
\end{array}\right.
$$

Следует отметить, что импеданс нагрузки должен быть согласован с сопротивлением массива $\left(\left|Z_{\text {Load }}\right| \sim N R_{J}\right)[24]$.

Численно решив систему уравнений (16) методом Рунге-Кутты 4-го порядка, среднюю поглощенную мощность для массива контактов можно расчитать по формуле (2). В начальный момент времени контакты считались не синхронизированными, т.е. начальные условия в системе (16) задавались произвольно. Численный анализ показал, что контакты в массиве могут синхронизироваться таким образом, что усиление, получаемое на каждом контакте массива, складывается с усилением других контактов, лишь при небольших емкостях резонатора, т.е. при $\beta \ll 1$, даже если изначально контакты в массиве были не синхронизированы. BAX и графики зависимости поглощенной мощности от постоянного тока для массива из $N=10$ контактов представлены на рис. 10.

Из рис. 10 видно, что при увеличении числа контактов поглощенная мощность (усиление) увеличивается пропорционально числу синхронизированных контактов в массиве.

На рис. 11 представлены ВАХ и график зависимости средней поглощенной мощности массива из 10 контактов для тех же значений параметров системы, что и на рис. 5.

Из сравнения рис. 11 и 5 видно, что область усиления вблизи субгармонической ступеньки, обнаруженная для одиночного контакта в резонаторе, отсутствует на гра- 


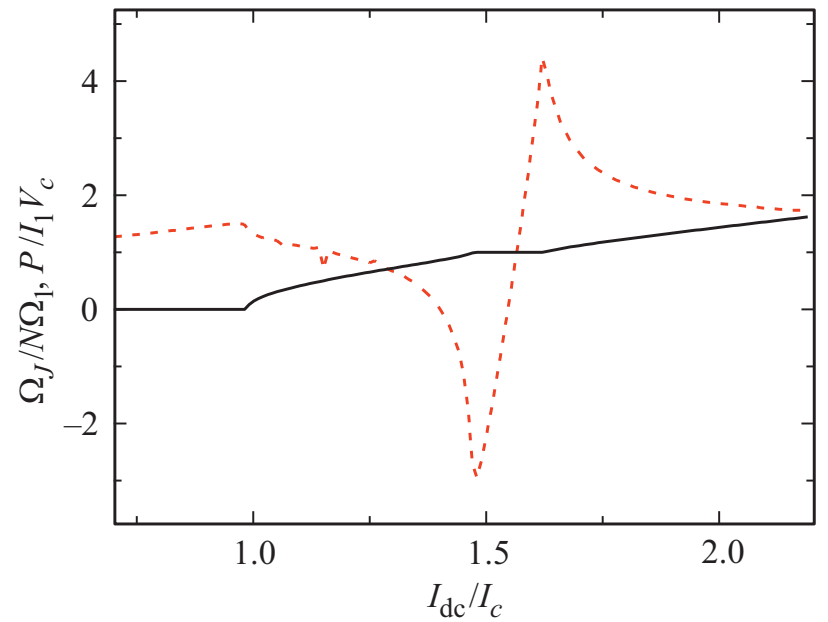

Рис. 10. BAX и график зависимости поглощенной мощности от постоянного тока для массива из 10 контактов в резонаторе. $\Omega_{1}=1.2 ; \Omega_{0}=2.4 ; I_{1}=0.3 I_{c} ; \beta=0.01$.
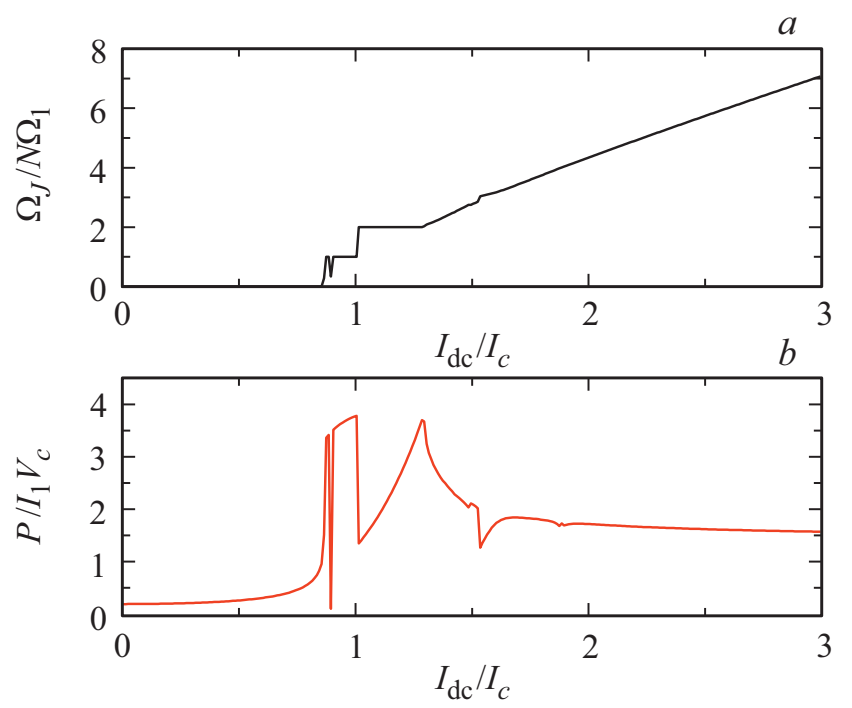

Рис. 11. a) BAX и $b$ ) график зависимости поглощенной мощности от постоянного тока для массива из 10 контактов в резонаторе. $\Omega_{1}=0.4 ; \Omega_{0}=0.8 ; I_{1}=0.3 I_{c} ; \beta=0.01$.

фике средней поглощенной мощности для одномерного массива при тех же параметрах системы, а вместо субгармонической ступеньки видим искажение первой ступеньки Шапиро.

\section{5. Заключение}

В рамках резистивной модели теоретически изучено влияние резонатора на ВАХ и параметрическое усиление точечного контакта Джозефсона. В рамках резистивной модели вычислена средняя поглощенная мощность внешнего монохроматического ВЧ сигнала для точечного контакта Джозефсона в резонаторе и построена ВАХ контакта в резонаторе во внешнем ВЧ поле. Найдены области усиления (отрицательной средней поглощенной мощности) внешнего излучения для контакта Джозефсона в резонаторе. Численный анализ показал, что при некоторых значениях параметров резонатора (в частности, емкости и частоты) области усиления системы могут располагаться вблизи субгармонических ступенек вольт-амперной характеристики. Тут же отметим, что для контакта во внешнем монохроматическом ВЧ поле область усиления располагается только вблизи первой ступеньки Шапиро. Наличие области усиления вблизи субгармонических ступенек ВАХ было подтверждено аналитически в рамках теории возмущений для кратных частот резонатора, внешнего ВЧ сигнала и джозефсоновской частоты. Также численный анализ показал, что вблизи субгармонических ступенек BAX точечные контакты Джозефсона, последовательно соединенные в одномерном массиве, не синхронизируются и область усиления вблизи субгармонической ступеньки для массива отсутствует.

\section{Приложение A}

Решим систему уравнений (6) с учетом (А.8). Функция в правой части уравнения (7) являеся периодической, поэтому удобно представить ее в виде ряда Фурье

$$
\begin{aligned}
\ddot{q} & +\Omega_{0}^{2} q=2 \Omega_{J} C \Omega_{0}^{2} \\
& +2 \Omega_{J} \Omega_{0}^{2} \sum_{k=1}^{\infty}\left(\Omega_{J}-\sqrt{1+\Omega_{J}^{2}}\right)^{k} \cos \left(k \Omega_{J} \tau\right),
\end{aligned}
$$

а частное решение уравнения искать в виде правой части, т. е. в виде ряда:

$$
q_{\text {part }}=Q_{0}+\sum_{k=1}^{\infty} Q_{k} \cos \left(k \Omega_{J} \tau\right) .
$$

Подставим частное решение (А.2) в уравнение (А.1)

$$
\begin{gathered}
-\Omega_{J}^{2} \sum_{k=1}^{\infty} k^{2} Q_{k} \cos \left(k \Omega_{J} \tau\right)+\Omega_{0}^{2} Q_{0}+\Omega_{0}^{2} \sum_{k=1}^{\infty} Q_{k} \cos \left(k \Omega_{J} \tau\right) \\
=2 \Omega_{J} \Omega_{0}^{2}+2 \Omega_{J} \Omega_{0}^{2} \sum_{k=1}^{\infty}\left(\Omega_{J}-\sqrt{1+\Omega_{J}^{2}}\right)^{k} \cos \left(k \Omega_{J} \tau\right)
\end{gathered}
$$

откуда найдем коэффициенты

$$
Q_{0}=2 \Omega_{J} ; \quad Q_{k}=2 \Omega_{J} \Omega_{0}^{2} \frac{\left(\Omega_{J}-\sqrt{1+\Omega_{J}^{2}}\right)^{k}}{\Omega_{0}^{2}-k^{2} \Omega_{J}^{2}} .
$$

Частное решение примет вид

$$
q_{\text {part }}=2 \Omega_{J}+2 \Omega_{J} \Omega_{0}^{2} \sum_{k=1}^{\infty} \frac{\left(\Omega_{J}-\sqrt{1+\Omega_{J}^{2}}\right)^{k}}{\Omega_{0}^{2}-k^{2} \Omega_{J}^{2}} \cos \left(k \Omega_{J} \tau\right) .
$$

Общее решение однородного уравнения для (7) очевидHO

$$
q_{\text {hom }}=A \cos \Omega_{0} \tau+B \sin \Omega_{0} \tau,
$$

$A$ и $B-$ константы интегрирования. 
Задав нулевые начальные условия $(q(0)=0$ и $\dot{q}(0)=0)$, можно найти значение постоянных интегрирования и окончательно записать решение уравнения (А.1) для заряда на конденсаторе в виде

$$
q(\tau)=2 \Omega_{J}-2 \Omega_{J} \cos \Omega_{0} \tau+2 \Omega_{J} \Omega_{0}^{2} \sum_{k=1}^{\infty} \frac{\left(\Omega_{J}-\sqrt{1+\Omega_{J}^{2}}\right)^{k}}{\Omega_{0}^{2}-k^{2} \Omega_{J}^{2}}\left[\cos \left(k \Omega_{J} \tau\right)-\cos \Omega_{0} \tau\right] .
$$

Решив первое уравнение системы (6) с учетом (А.7) и (10), получим

$$
\begin{aligned}
\dot{\tilde{\varphi}}_{\text {res }}(\tau)= & -2 \beta \Omega_{J} \Omega_{0}\left[1+\Omega_{0}^{2} \sum_{k=1}^{\infty} \frac{\left(\Omega_{J}-\sqrt{1+\Omega_{J}^{2}}\right)^{k}}{\Omega_{0}^{2}-k^{2} \Omega_{J}^{2}}\right] \sin \Omega_{0} \tau+2 \beta \Omega_{J}^{2} \Omega_{0}^{2} \sum_{k=1}^{\infty} \frac{k\left(\Omega_{J}-\sqrt{1+\Omega_{J}^{2}}\right)^{k}}{\Omega_{0}^{2}-k^{2} \Omega_{J}^{2}} \sin \left(k \Omega_{J} \tau\right) \\
& -\frac{\beta \Omega_{J} \sin \Omega_{J} \tau}{\left(\sqrt{1+\Omega_{J}^{2}}+\cos \Omega_{J} \tau\right)^{2}}\left\{-2 \Omega_{J} \sqrt{1+\Omega_{J}^{2}}\left[1+\Omega_{0}^{2} \sum_{k=1}^{\infty} \frac{\left(\Omega_{J}-\sqrt{1+\Omega_{J}^{2}}\right)^{k}}{\Omega_{0}^{2}-k^{2} \Omega_{J}^{2}}\right] \cos \Omega_{0} \tau\right. \\
& +2 \Omega_{J} \Omega_{0}^{2} \sqrt{1+\Omega_{J}^{2}} \sum_{k=1}^{\infty} \frac{\left(\Omega_{J}-\sqrt{1+\Omega_{J}^{2}}\right)^{k}}{\Omega_{0}^{2}-k^{2} \Omega_{J}^{2}} \cos \left(k \Omega_{J} \tau\right)-\Omega_{J} \Omega_{0}\left[1+\Omega_{0}^{2} \sum_{k=1}^{\infty} \frac{\left(\Omega_{J}-\sqrt{1+\Omega_{J}^{2}}\right)^{k}}{\Omega_{0}^{2}-k^{2} \Omega_{J}^{2}}\right] \\
& \times\left[\frac{\cos \left[\left(\Omega_{0}+\Omega_{J}\right) \tau\right]}{\Omega_{0}+\Omega_{J}}+\frac{\cos \left[\left(\Omega_{0}-\Omega_{J}\right) \tau\right]}{\Omega_{0}-\Omega_{J}}\right]+\Omega_{J} \Omega_{0}^{2} \sum_{k=2}^{\infty} \frac{k\left(\Omega_{J}-\sqrt{1+\Omega_{J}^{2}}\right)^{k}}{\Omega_{0}^{2}-k^{2} \Omega_{J}^{2}} \\
& \left.\times\left[\frac{\cos \left[(k+1) \Omega_{J} \tau\right]}{k+1}+\frac{\cos \left[(k-1) \Omega_{J} \tau\right]}{k-1}\right]-\frac{\Omega_{0}^{2}}{2}\left(\frac{\Omega_{J}-\sqrt{1+\Omega_{J}^{2}}}{\Omega_{0}^{2}-\Omega_{J}^{2}}\right) \cos ^{2} \Omega_{J} \tau\right\} .
\end{aligned}
$$

Возьмем слабый внешний сигнал в виде $\tilde{i}_{1}=\exp \left(i \Omega_{1} \tau\right)$. Найдем $R e Z_{r e s}$ по формуле (13). Используя формулу Эйлера и разложение в ряд Фурье множителя перед фигурной скобкой в соотношении (А.8)

$$
\frac{\beta \Omega_{J} \sin \Omega_{J} \tau}{\left(\sqrt{1+\Omega_{J}^{2}}+\cos \Omega_{J} \tau\right)^{2}}=-\frac{2 \beta}{\Omega_{J}} \sum_{n=1}^{\infty} n\left(\Omega_{J}-\sqrt{1+\Omega_{J}^{2}}\right)^{n} \sin \left(n \Omega_{J} \tau\right),
$$

для $\operatorname{Re} \frac{\dot{\hat{\varphi}}_{\text {res }}}{\dot{i}_{1}}$ получим

$$
\begin{aligned}
\operatorname{Re} \frac{\dot{\tilde{\varphi}}_{\text {res }}}{\tilde{i}_{1}}= & -\beta \Omega_{J} \Omega_{0}\left[1+\Omega_{0}^{2} \sum_{k=1}^{\infty} \frac{\left(\Omega_{J}-\sqrt{1+\Omega_{J}^{2}}\right)^{k}}{\Omega_{0}^{2}-k^{2} \Omega_{J}^{2}}\right]\left[\sin \left[\left(\Omega_{0}-\Omega_{1}\right) \tau\right]+\sin \left[\left(\Omega_{0}+\Omega_{1}\right) \tau\right]\right] \\
& +\beta \Omega_{J}^{2} \Omega_{0}^{2} \sum_{k=1}^{\infty} \frac{k\left(\Omega_{J}-\sqrt{1+\Omega_{J}^{2}}\right)^{k}}{\Omega_{0}^{2}-k^{2} \Omega_{J}^{2}}\left[\sin \left[\left(k \Omega_{J}-\Omega_{1}\right) \tau\right]+\sin \left[\left(k \Omega_{J}+\Omega_{1}\right) \tau\right]\right]+\frac{2 \beta}{\Omega_{J}} \sum_{n=1}^{\infty} n\left(\Omega_{J}-\sqrt{1+\Omega_{J}^{2}}\right)^{n} \\
& \times\left\{-\frac{\Omega_{J}}{2} \sqrt{1+\Omega_{J}^{2}}\left[1+\Omega_{0}^{2} \sum_{k=1}^{\infty} \frac{\left(\Omega_{J}-\sqrt{1+\Omega_{J}^{2}}\right)^{k}}{\Omega_{0}^{2}-k^{2} \Omega_{J}^{2}}\right]\left[\sin \left[\left(n \Omega_{J}+\Omega_{0}-\Omega_{1}\right) \tau\right]+\sin \left[\left(n \Omega_{J}-\Omega_{0}-\Omega_{1}\right) \tau\right]\right.\right. \\
& \left.+\sin \left[\left(n \Omega_{J}-\Omega_{0}+\Omega_{1}\right) \tau\right]+\sin \left[\left(n \Omega_{J}+\Omega_{0}+\Omega_{1}\right) \tau\right]\right]+\frac{\Omega_{J} \Omega_{0}^{2}}{2} \sqrt{1+\Omega_{J}^{2}} \sum_{k=1}^{\infty} \frac{\left(\Omega_{J}-\sqrt{1+\Omega_{J}^{2}}\right)^{k}}{\Omega_{0}^{2}-k^{2} \Omega_{J}^{2}} \\
& \left.\left.\left.\left.\times\left[\sin \left[(n+k) \Omega_{J}-\Omega_{1}\right) \tau\right]+\sin \left[(n-k) \Omega_{J}-\Omega_{1}\right) \tau\right]+\sin \left[(n-k) \Omega_{J}+\Omega_{1}\right) \tau\right]+\sin \left[(n+k) \Omega_{J}+\Omega_{1}\right) \tau\right]\right]
\end{aligned}
$$




$$
\begin{aligned}
& -\frac{\Omega_{J} \Omega_{0}}{4}\left[1+\Omega_{0}^{2} \sum_{k=1}^{\infty} \frac{\left(\Omega_{J}-\sqrt{1+\Omega_{J}^{2}}\right)^{k}}{\Omega_{0}^{2}-k^{2} \Omega_{J}^{2}}\right]\left[\frac{\sin \left[\left((n+1) \Omega_{J}+\Omega_{0}-\Omega_{1}\right) \tau\right]}{\Omega_{J}+\Omega_{0}}+\frac{\sin \left[\left((n-1) \Omega_{J}-\Omega_{0}-\Omega_{1}\right) \tau\right]}{\Omega_{J}+\Omega_{0}}\right. \\
& +\frac{\sin \left[\left((n-1) \Omega_{J}-\Omega_{0}+\Omega_{1}\right) \tau\right]}{\Omega_{J}+\Omega_{0}}+\frac{\sin \left[\left((n+1) \Omega_{J}+\Omega_{0}+\Omega_{1}\right) \tau\right]}{\Omega_{J}+\Omega_{0}}+\frac{\sin \left[\left((n-1) \Omega_{J}+\Omega_{0}-\Omega_{1}\right) \tau\right]}{\Omega_{J}-\Omega_{0}} \\
& \left.+\frac{\sin \left[\left((n+1) \Omega_{J}-\Omega_{0}-\Omega_{1}\right) \tau\right]}{\Omega_{J}-\Omega_{0}}+\frac{\sin \left[\left((n+1) \Omega_{J}-\Omega_{0}+\Omega_{1}\right) \tau\right]}{\Omega_{0}-\Omega_{J}}+\frac{\sin \left[\left((n-1) \Omega_{J}+\Omega_{0}+\Omega_{1}\right) \tau\right]}{\Omega_{0}-\Omega_{J}}\right] \\
& +\frac{\Omega_{J} \Omega_{0}^{2}}{4} \sum_{k=2}^{\infty} \frac{k\left(\Omega_{J}-\sqrt{1+\Omega_{J}^{2}}\right)^{k}}{\Omega_{0}^{2}-k^{2} \Omega_{J}^{2}}\left[\frac{\sin \left[\left((n+k+1) \Omega_{J}-\Omega_{1}\right) \tau\right]}{k+1}+\frac{\sin \left[\left((n-k-1) \Omega_{J}-\Omega_{1}\right) \tau\right]}{k+1}\right. \\
& +\frac{\sin \left[\left((n-k-1) \Omega_{J}+\Omega_{1}\right) \tau\right]}{k+1}+\frac{\sin \left[\left((n+k+1) \Omega_{J}+\Omega_{1}\right) \tau\right]}{k+1}+\frac{\sin \left[\left((n+k-1) \Omega_{J}-\Omega_{1}\right) \tau\right]}{k-1} \\
& \left.+\frac{\sin \left[\left((n-k+1) \Omega_{J}-\Omega_{1}\right) \tau\right]}{k-1}+\frac{\sin \left[\left((n-k+1) \Omega_{J}+\Omega_{1}\right) \tau\right]}{k-1}+\frac{\sin \left[\left((n+k-1) \Omega_{J}+\Omega_{1}\right) \tau\right]}{k-1}\right] \\
& -\frac{\Omega_{0}^{2}}{8}\left(\frac{\Omega_{J}-\sqrt{1+\Omega_{J}^{2}}}{\Omega_{0}^{2}-\Omega_{J}^{2}}\right)\left[\sin \left[(n+2) \Omega_{J} \tau\right]+\sin \left[(n-2) \Omega_{J} \tau\right]+\sin \left[\left((n+2) \Omega_{J}+\Omega_{1}\right) \tau\right]\right. \\
& \left.\left.+\sin \left[\left((n-2) \Omega_{J}+\Omega_{1}\right) \tau\right]+\sin \left[\left((n-2) \Omega_{J}-\Omega_{1}\right) \tau\right]+\sin \left[\left((n+2) \Omega_{J}-\Omega_{1}\right) \tau\right]\right]\right\} .
\end{aligned}
$$

Среднее значение $\left\langle\operatorname{Re} \frac{\dot{\tilde{\varphi}}_{1}}{\tilde{i}_{1}}\right\rangle$, взятое по общему периоду в выражении (А.10), отлично от нуля лишь в случае $\Omega_{0} \rightarrow k \Omega_{J}$, где $k=1,2,3, \ldots$. Усредняя (А.10) по общему периоду и осуществляя предельный переход при $\Omega_{0} \rightarrow k \Omega_{J}$ для случая $\Omega_{0}=\Omega_{1}=k \Omega_{J}(k>1)$, получим выражение (14) для $\operatorname{Re} Z_{\text {res. }}$. Для соотношения частот $\Omega_{0}=n \Omega_{1}=k \Omega_{J}$, где $n=2,3, \ldots$ и $k>1$, усредняя (А.10) по общему периоду и осуществляя предельный переход при $\Omega_{0} \rightarrow k \Omega_{J}$, получим выражение (15) для $\operatorname{Re} Z_{\text {res }}$.

\section{Список литературы}

[1] B.D. Josephson. Rev. Mod. Phys. 36, 216 (1964).

[2] Л.Г. Асламазов, А.И. Ларкин, Письма в ЖЭТФ 9, 150 (1969).

[3] К.К. Лихарев, В.К. Семенов. Радиотехника и электроника 11, 2167 (1971).

[4] А.Н. Выставкин, В.Н. Губанков, Л.С. Кузьмин, К.К. Лихарев, В.В. Митулин. Радиотехника и электроника 17, 896 (1971).

[5] А.Н. Выставкин, В.Н. Губанков, Г.Ф. Лещенко, К.К. Лихарев, В.В. Митулин. Радиотехника и электроника 15, 2404 (1970).

[6] H. Kanter, F.L. Vernon. J. Appl. Phys. 43, 3174 (1972).

[7] E.D. Thompson. J. Appl. Phys. 44, 5587 (1973).

[8] F. Auracher, T. Van Duzer. J. Appl. Phys. 44, 2, 848 (1973).
[9] А.Н. Выставкин, В.Н. Губанков, Л.С. Кузьмин, К.К. Лихарев, В.В. Мигулин, А.Н. Спицын. Письма в ЖЭТФ 17, 284 (1973).

[10] H. Kanter. Appl. Phys. Lett. 23, 350 (1973).

[11] Fengbin Song, Franz Muller, Thomas Scheller, Alexei Semenov, Ming He, Lan Fang, Heinz-Wilhelm Hubers, Alexander M. Klushin. Appl. Phys. Lett. 98, 142506 (2011).

[12] Masashi Tachiki, Krsto Ivanovic, Kazuo Kadowaki, Tomio Koyama. Phys. Rev. B 83, 014508 (2011).

[13] S. Shapiro. Phys. Rev. Lett. 11, 80 (1963).

[14] Ma.A. Gaafar, Yu.M. Shukrinov, A. Foda. J. Phys.: Conf. Ser. 393, 012021 (2012).

[15] P.W. Anderson, A.H. Dayem. Phys. Rev. Lett. 13, 195 (1964).

[16] C.A. Hamilton, Jr. E.G. Johnson. Phys. Lett. A. 41, 393 (1972).

[17] L.E. Hasselberg, M.T. Levinsen, M.R. Samuelsen. Rev. Phys. Appl. 9, 157(1974).

[18] P.E. Gregers-Hansen, G.R. Pickett. Rev. Phys. Appl. 9, 145 (1974).

[19] L.E. Hasselberg, M.T. Levinsen, M.R. Samueisen. J. Low Temperat. Phys. 21, 567 (1975).

[20] P. Seidel, M. Siegel, E. Heinz. Physica C 180, 284 (1991).

[21] M. Darula, T. Doderer, S. Beuven. Supercond. Sci. Technol. 12, R1-R25 (1999).

[22] G. Filatrella, N.F. Pedersen, K. Wiesenfeld. Phys. Rev. E 61, 2513 (2000).

[23] Juan A. Acebron, L.L. Bonilla, Conrad J. Perez Vicente, Felix Ritort, Renato Spigler. Rev. Mod. Phys. 77, 138 (2005). 
[24] A.K. Jain, K.K. Likharev, J.E. Lukens, J.E. Sauvageau. Phys. Rep. 109, 309 (1984).

[25] J.W. Swift, S.H. Strogatz, K. Wiesenfeld. Physica D 55, 239 (1992).

[26] P. Hadley, M.R. Beasley. Appl. Phys. Lett. 50, 621 (1987).

[27] P. Hadley, M.R. Beasley, K. Wiesenfeld. Phys. Rev. B 38, 8712 (1988).

[28] K. Wiesenfeld, J.W. Swiftt. Phys. Rev. E 51, 1020 (1995).

[29] V. Vlasov, A. Pikovsky. Phys. Rev. E 88, 022908 (2013).

[30] Shi-Zeng Lin, Xiao Hu, Lev Bulaevskii. Phys. Rev. B 84, 104501 (2011).

Редактор Т.Н. Василевская 